\title{
Research on Decision Support System of Vehicle Maintenance Support
}

\author{
Songbai $\mathrm{He}^{1, \mathrm{a}}$, Kai Kang ${ }^{2, \mathrm{~b}}$, Jingxin $\mathrm{Li}^{3, \mathrm{c}}$ \\ ${ }^{1}$ School of Economics and Management, Hebei University of Technology, Tianjin 300132, China \\ ${ }^{2}$ School of Economics and Management, Hebei University of Technology, Tianjin 300132, China \\ ${ }^{1,3}$ Academy of Military Transportation, Tianjin 300161, China \\ ahsbcool@163.com, bkkang2000@sina.com, c13207630891@163.com
}

\section{Keywords: Decision Support System; Vehicle Maintenance Support; Model Base}

Abstract:Vehicle maintenance support decision is critical in emergency actions. Based on the analysis of vehicle maintenance support elements and processes, this paper builds the architecture of vehicle maintenance support decision support system, and establishes a function module which can accurately describe vehicle maintenance support decision. It will provide basis for vehicle maintenance support decision optimization.

\section{Introduction}

Vehicle maintenance support asks for rapid response, accurate support and the lowest consumption. That is, to minimize the consumption of resources, so that vehicles get timely, effective and economical maintenance support, so as to give full play to the effectiveness of vehicles. This requires that vehicle maintenance support realize digitization, automation, networking and integration as soon as possible. Therefore, it is particularly important to establish a decision support system which can meet the requirements of both structured and semi-structured, unstructured decision, and comprehensive utilization of data, information and knowledge ${ }^{[1]}$.

\section{Related Factors of Vehicle Maintenance Support}

Maintenance Task. Maintenance task is to give the maintenance team the mission to go out for maintain. The main task of vehicle maintenance is to keep the vehicle in good technical condition. Its contents are shown in Figure 1. Among them: a maintenance task has only one unique identifier of the mission, but it may require maintenance of multiple fault points in different locations, for the same fault may be corresponding to a plurality of different maintenance scheme. One of the most important functions about the vehicle maintenance decision support system is to optimize the maintenance of multiple fault points in a maintenance task, so as to achieve the optimal maintenance time, the shortest path and the least consumption.

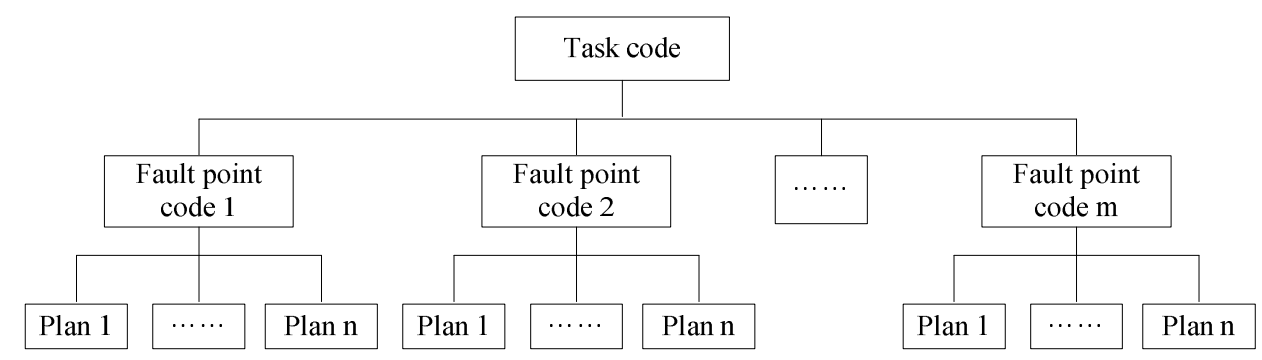

Fig. 1 Schematic diagram of maintenance task 
Maintenance Team. Maintenance team refers to a temporarily formed collection of the maintenance personnel, vehicles, maintenance spare parts and maintenance equipment after receiving the maintenance task. When the task is completed, it is automatically canceled, and the resources are stored in the warehouse. When there is a maintenance task, the system will optimize the maintenance personnel, vehicles, spare parts, and set up a maintenance team. And give the maintenance task a unique task code, at this time the maintenance team is in the state of dispatch. Once the task is completed, the maintenance team will automatically cancel. The name of the maintenance personnel will enter the static resource library, put the remaining spare parts into the warehouse, and add the corresponding spare parts name to the material storage table, and release the corresponding resources into storage. When you have a maintenance task again, you can continue to call the freed resources.

Fault Point. Fault point refers to the location where the vehicle can't be used properly and need to be repaired by the dispatch maintenance personnel. When the maintenance unit receives the maintenance task, a temporary record of the fault point is established. The degree of urgency mainly depends on the length of time required to repair the damaged vehicles at the fault point. Requirements in the shorter period of time to repair the damaged vehicles, indicating that the urgency of the fault point is higher. It takes time for the maintenance team from forming to reach the fault point, and in the process, the fault point will communicate at any time before the maintenance team arrives. In order to avoid repeated dispatch of maintenance team, the fault point will be set to dispatch, wait and end three states. Dispatch refers to the maintenance unit has sent the maintenance team, wait means maintenance team has not yet formed due to lack of the maintenance personnel, vehicles, equipment or tools, end refers to the vehicles in the fault point have been completely repaired. When the maintenance unit arranges the maintenance team, as long as the fault point has the corresponding task code, the system will prompt the operator that the maintenance team has been arranged.

Static Resources. Static resources refer to the collection of available maintenance personnel, vehicles, equipment, tools and so on, which is used by maintenance unit to complete maintenance tasks. Static resources are an important part of decision support system for maintenance support. The unreasonable determination of static security resources often leads to serious shortage and serious backlog of some resources, which will directly affect the readiness and maneuverability of vehicles. Lack of maintenance resources support or lack of security will also seriously affect training and task completion. Therefore, the determination and optimization of the vehicle maintenance support resources, which makes the maintenance support capability to maximize the transformation into direct effectiveness, is a problem that the decision makers must consider.

\section{Analysis of Vehicle Maintenance Support Process}

Analysis of Vehicle Maintenance Support Task Dispatch Process. The vehicle maintenance support task dispatch process is shown in Figure 2. The decision support system of vehicle maintenance support is mainly to make decision on the information that is transmitted from the fault point and processed by the management information system and expert system. If the decision support system is used as the master control system, an interface can be designed to deal with the information of the fault point. The information should include: mission code, unit code, fault type, 
the location of the fault point and the urgency of the maintenance. It should be noted that, due to emergency situations require more maintenance of vehicles, a maintenance team can't be sent immediately to a task. Therefore, in the situation that the fault point requires that the urgency of maintenance is not particularly high, it is necessary to specify a time interval for the task arrangement. The task processing of the decision support system for vehicle maintenance support is to sort the tasks in the time interval according to the urgency and the mileage of the maintenance points. Task has high urgency should be discharged in the front, and the adjacent fault point should be completed by one maintenance team as far as possible ${ }^{[2]}$.

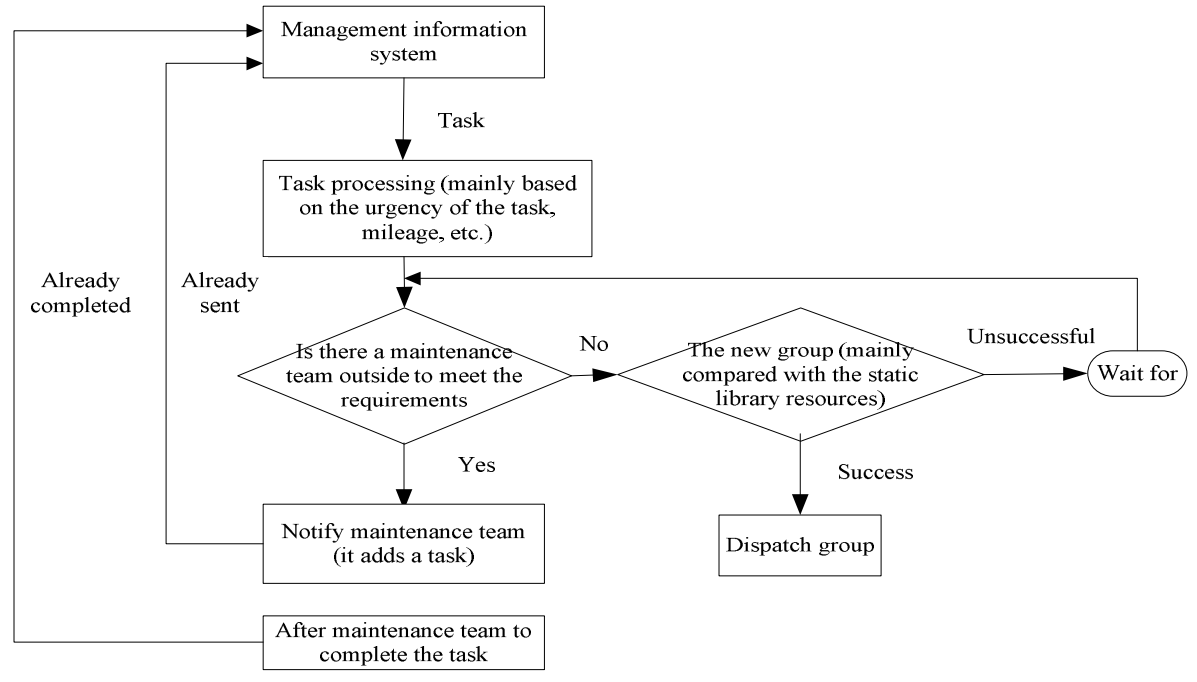

Fig. 2 Flow chart of vehicle maintenance support task dispatch

Analysis of Vehicle Maintenance Support Path Calculation Process. In the process of decision support for vehicle maintenance support, one of the most important links is to calculate the mileage between points. In this system a very important step is to map the points in the computer language into the database, and show the intersection in a special form.

(1)Path Initialization. The points on the map are written into the database in computer language. Each point on the graph is represented by a node, and multiple nodes form node table. The distance between two points is represented by a line, and multiple lines form line table. The specific mileage form odometer. Node table, line table, and odometer are combined into a structure chart. The node table includes node coding, node name, coordinate $\mathrm{X}$, coordinate $\mathrm{Y}$, node mark and so on. The line table includes line coding, line name, line grade and so on. The line odometer includes line coding, node coding, starting mileage, etc. It is shown in Figure 3.

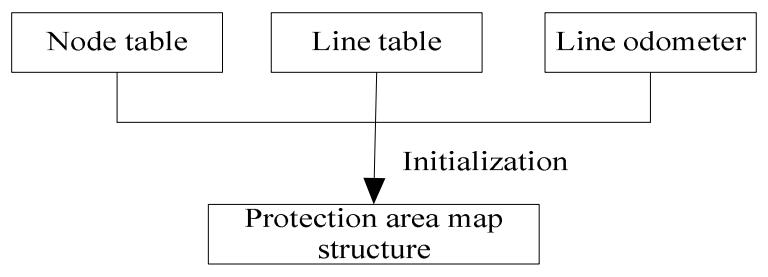

Fig. 3 Regional structure of vehicle maintenance support

(2) When the unit receive the transport task, if it is needed that vehicle support points belong to the support regional map location, its journey can be calculated directly from the distance between two points. If the site is not within the support regional, add the node as a midway point into the 
database, and calculate its distance. If you have to travel through a section of the road, consider the road as the center, and calculate the nearest route $^{[3]}$. The calculation process is shown in Figure 4.

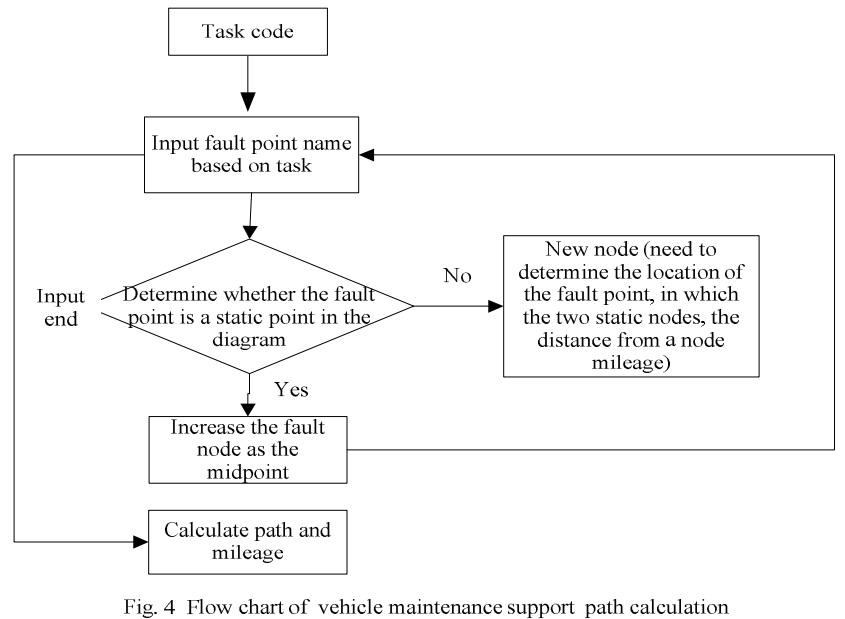

\section{Design on Decision Support System of Vehicle Maintenance Support}

This system is mainly composed of five functional modules, namely, user interaction module, data communication module, data management module, model management module and transportation decision module, the structure is shown in Figure 5. This paper focuses on the knowledge base, model base and database of the data management module ${ }^{[4]}$.

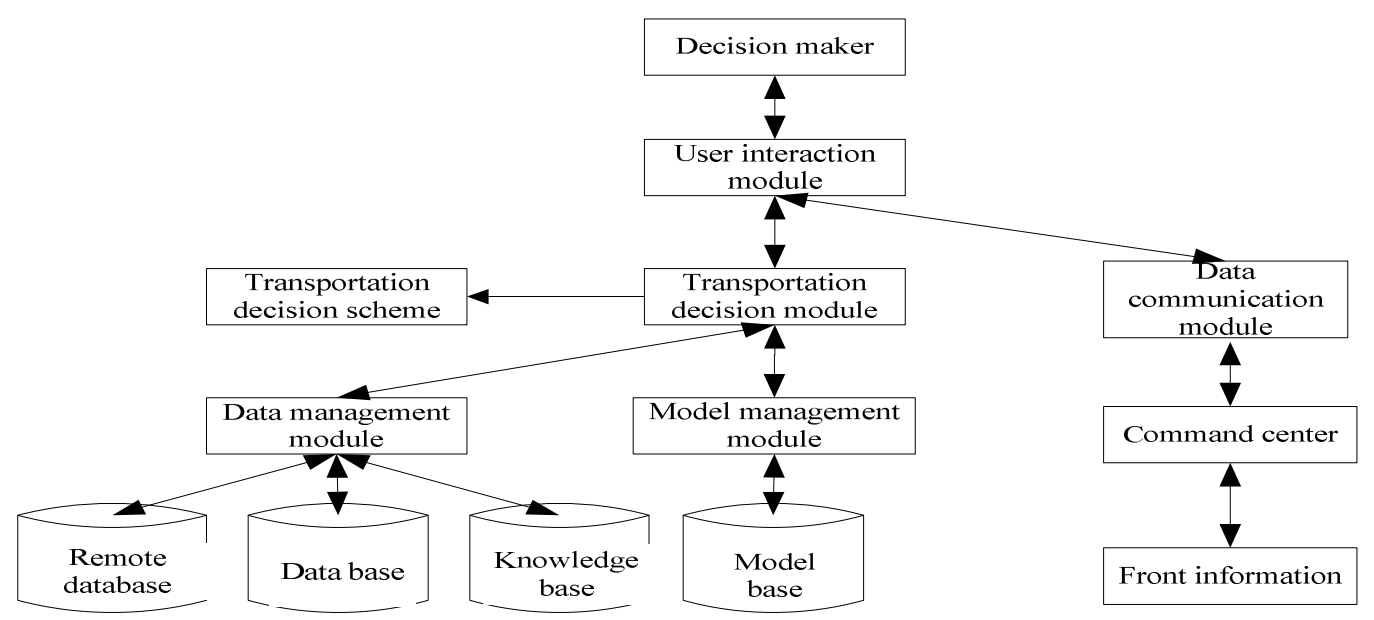

Fig. 5 Function module diagram of vehicle maintenance support decision system

Knowledge Base. Knowledge base is source of intelligence for vehicle maintenance support decision system, it contains two sub: fact base and rule base. The fact base is the domain knowledge that is entered by experts and the fact that it is summed up by the maintenance support decision, the structure is shown in Figure 6, including fact numbers and factual contents. The rule base is a record that is input by the expert or added by the system according to the previous vehicle maintenance support decision, each record in the rule base contains a rule, its structure is shown in Figure 7.

\begin{tabular}{|l|l|}
\hline Fact number & Factual content \\
\hline
\end{tabular}

Fig. 6 Record structure of vehicle maintenance support 


\begin{tabular}{|c|c|c|c|c|}
\hline Rule number & $\begin{array}{c}\text { Conditional fact } \\
\text { number }\end{array}$ & $\begin{array}{c}\text { Conditional fact } \\
\text { number }\end{array}$ & $\ldots \ldots \ldots$ & $\begin{array}{c}\text { Conclusion fact } \\
\text { number }\end{array}$ \\
\hline
\end{tabular}

Fig. 7 Record structure of vehicle maintenance support rule base

Model Base. The core part of vehicle maintenance decision support system, the realization of the decision support function of vehicle maintenance is mainly obtained through the model calculation, its model is shown in Figure 8. Mainly includes the following aspects: fault analysis and decision, maintenance personnel decision, maintenance spare parts decision, maintenance resource transportation decision and so on. Some constraints between these models are interrelated and mutually restricted. Therefore, in the process of decision problem solving, it is possible to make use of the cooperation among multiple models to solve a decision problem ${ }^{[5]}$.

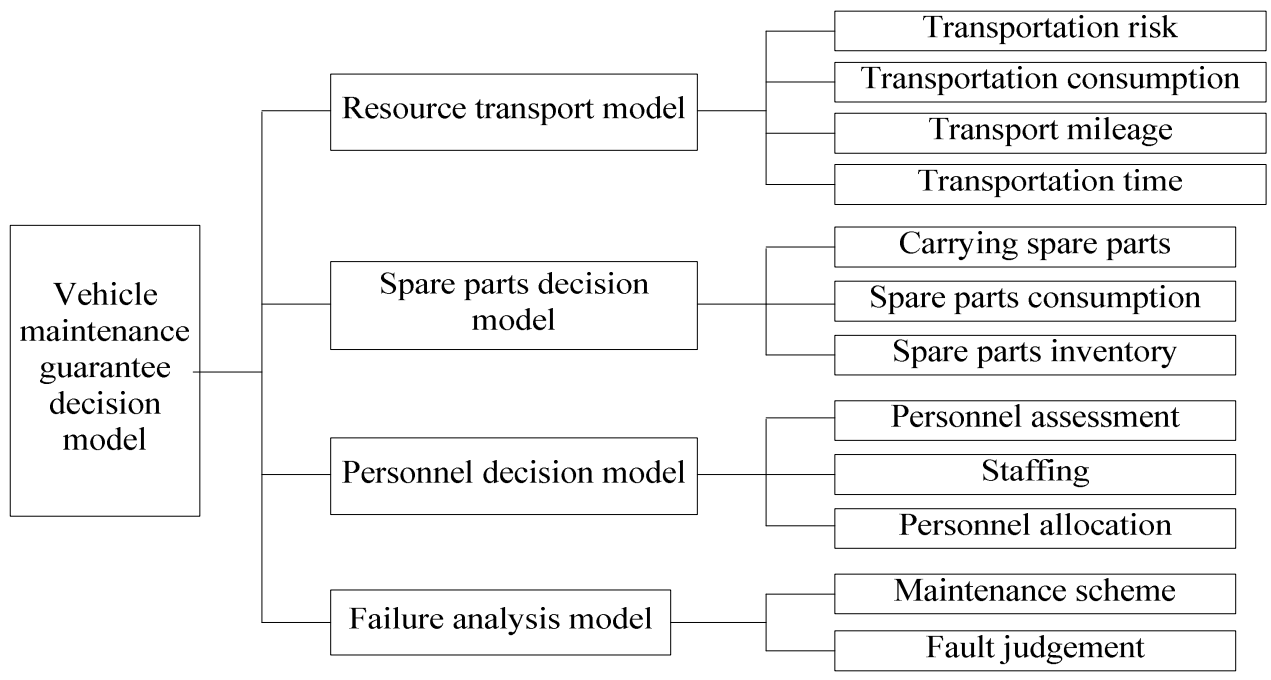

Fig. 8 Decision model of vehicle maintenance support

Data Base. Vehicle maintenance decision support system database includes the following aspects:

(1)Vehicle basic database. Some information about the basic situation of the vehicle, mainly includes: name, model, type, manufacturer, date of manufacture and the reliability, maintainability, security, mobility, etc. of the vehicle.

(2) Vehicle use database. Vehicle usage information, mainly includes: use unit, use time, intensity of use, use environment, service time and so on.

(3) Vehicle fault database. Vehicle fault repair and occurrence of failure probability and other relevant information, mainly include: type of fault, status of failure, and failure time, etc.

(4) Maintenance spare parts database. Reflect the variety, quantity and consumption of vehicle spare parts.

(5) Maintenance personnel database. Reflect the number of vehicle maintenance support personnel, professional conditions, etc.

(6) Cost database. Vehicle maintenance cost budget, get and paid, and actual balance of payments.

(7) Regional geographic information database. Store related map information, reflect the geographical location, topography and so on. 
8Regional road information database. Store information about road conditions in the relevant security area, mainly include: the maximum speed of the road and the speed of each period of time and so on.

\section{Conclusions}

Based on the modeling analysis of vehicle maintenance support elements and processes, this paper builds an architecture of vehicle maintenance decision support system.

(1) Using the modern decision support technology, the complex vehicle maintenance support links are decomposed according to the module, and the related model is used according to the needs to solve a sub-problem and achieve the solution of complex problems.

(2)Focusing on the importance of vehicle maintenance support, the models of maintenance tasks, maintenance teams, fault points and static resources are established, and the principle of operation is established, which is helpful to improve the efficiency of vehicle maintenance support.

(3) In order to automate the vehicle maintenance support decision, the process of vehicle maintenance support is standardized according to the module, and the corresponding knowledge base, model base and database are built, and the multi-model cooperative operation is realized through the vehicle maintenance decision support system.

\section{References}

[1]Alvina G.H. Kek, Ruey Long Cheu, Qiang Meng,Chau Ha Fung. A decision support system for vehicle relocation operations in carsharing systems[J]. Transportation Research Part E:Logistics and Transportation Review, 2009, 45(1):149-158.

[2]Jong-Ho Shin,Hong-Bae Jun,Cedric Catteneo Degradation mode and criticality analysis based on product usage data[J].The International Journal of Advanced Manufacturing Technology, 2015,78(9-12):1727-1742.

[3]Amie Wilson,Sarah Hillman,Mikey Rosato,John Skelton,Anthony Costello. A systematic review and thematic synthesis of qualitative studies on maternal emergency transport in low- and middle-income countries[J].International Journal of Gynecology and Obstetrics,2013,122(3):192-201.

[4]Susana Ferreiroa,sferreiro@tekniker.es,Aitor Arnaiza,Basilio Sierrab,Itziar Irigoienb. Application of Bayesian networks in prognostics for a new Integrated Vehicle Health Management concept[J]. Expert Systems with Applications,2012,39(7):6402-6418.

[5]Ahmad T. Mayyas, Ala Qattawi, Abdel Raouf Mayyas, Mohammed A. Omar, momar@clemson.edu. Life cycle assessment-based selection for a sustainable lightweight body-in-white design[J]. Energy,2012,39(1):412-425. 